\title{
Development of CRTEIL and CETRIZ, Cre-loxP-Based Systems, Which Allow Change of Expression of Red to Green or Green to Red Fluorescence upon Transfection with a Cre-Expression Vector
}

\author{
Masato Ohtsuka, ${ }^{1}$ Takayuki Warita, ${ }^{1}$ Takayuki Sakurai, ${ }^{2}$ Satoshi Watanabe, ${ }^{3}$ \\ Hidetoshi Inoko, ${ }^{1}$ and Masahiro Sato ${ }^{4}$ \\ ${ }^{1}$ Division of Basic Molecular Science and Molecular Medicine, School of Medicine, Tokai University, Bohseidai, \\ Isehara, Kanagawa 259-1193, Japan \\ ${ }^{2}$ Department of Organ Regeneration, Graduate School of Medicine, Shinshu University, 3-1-1 Asahi, Matsumoto, \\ Nagano 390-8621, Japan \\ ${ }^{3}$ Department of Developmental Biology, National Institute of Agrobiological Sciences, 2 Ikenodai, Tsukuba, Ibaraki, \\ 305-0901, Japan \\ ${ }^{4}$ Section of Gene Expression Regulation, Frontier Science Research Center, Kagoshima University, 1-21-20 Korimoto, \\ Kagoshima 890-0065, Japan
}

Correspondence should be addressed to Masahiro Sato, masasato@ms.kagoshima-u.ac.jp

Received 12 September 2008; Accepted 13 January 2009

Recommended by Vladimir Larionov

We developed Cre-loxP-based systems, termed CRTEIL and CETRIZ, which allow gene switching in a noninvasive manner. Single transfection with pCRTEIL resulted in predominant expression of red fluorescence. Cotransfection with pCRTEIL and Creexpression plasmid (pCAG/NCre) caused switching from red to green fluorescence. Similarly, cotransfection with pCETRIZ and pCAG/NCre resulted in change of green to red fluorescence. These noninvasive systems will be useful in cell lineage analysis, since descendants of cells exhibiting newly activated gene expression can be continuously monitored in noninvasive fashion.

Copyright (C) 2009 Masato Ohtsuka et al. This is an open access article distributed under the Creative Commons Attribution License, which permits unrestricted use, distribution, and reproduction in any medium, provided the original work is properly cited.

\section{Introduction}

The bacteriophage P1 Cre recombinase, a $38-\mathrm{kDa}$ protein, recognizes specific 34 -bp sequences called loxP sites and catalyzes site-specific recombination between two loxP sites $[1,2]$. The Cre-loxP system has been considered an important tool for manipulation of genomic sequences and gene expression including tissue-specific activation or inactivation of genes as well as cell-type-specific gene targeting [3]. Araki et al. [4] first utilized this system for manipulation of mouse preimplantation embryos. They constructed a CAG-CAT-Z transgene, in which expression of the floxed chloramphenicol acetyltransferase (CAT) gene occurs under control of the ubiquitously active cytomegalovirus enhancer/chicken $\beta$ actin promoter (CAG) [5]. Expression of the lacZ gene coding for bacterial $\beta$-galactosidase protein, which is located downstream of the floxed CAT sequence in the CAGCAT-Z transgene, does not occur in the absence of Cre recombinase protein. When Cre-expression vector DNA was injected into the pronuclei of fertilized eggs carrying the CAG-CAT-Z transgene, expression of lacZ protein was induced, indicating that expression of the lacZ gene is induced after removal of the floxed CAT sequence by Cre recombinase. Once such recombination occurs, expression of the lacZ gene in descendant cells will continue throughout embryogenesis.

Using a strategy similar to that of Araki et al. [4], we previously produced a double-reporter mouse line carrying a pCETZ-17 transgene, which consists of CAG, floxed DNA sequence (containing enhanced green fluorescent protein 
(EGFP) cDNA), and the lacZ gene [6]. This Cre-loxP-based system was useful for cell lineage analysis in mice. For example, when one blastomere of the 2-cell embryos derived from CETZ-17 transgenic mice was microinjected with Cre-expression vector (pCAG/NCre-5) [7], its descendant exhibited Cre-mediated recombination in the integrated transgenes and also exhibited lacZ activity. However, the offspring of the other blastomere, into which no injection was performed, remained silent for lacZ activity. In this case, histochemical detection of lacZ activity is always associated with fixation of tissues and subsequent staining with X-Gal, a substrate for lacZ, or by immunodetection using anti- $\beta$ galactosidase antibodies. This procedure often hampers serial microscopic observation of cells expressing newly activated gene products, and some tissues (i.e., adult ovaries, oviducts, and epididymides) are rich in endogenous activity for lacZ (unpublished data).

In this study, we constructed two new plasmids, pCRTEIL and pCETRIZ, as double-reporter transgenes. pCRTEIL contains CAG, floxed sequence (HcRed1 cDNA and CAT gene), EGFP cDNA, internal ribosomal entry site (IRES) from the encephalomyocarditis virus, and firefly luciferase (luc) cDNA. pCETRIZ contains CAG, floxed EGFP cDNA and CAT gene, HcRed1 cDNA, IRES, and lacZ gene. For example, cells carrying pCRTEIL exhibit HcRed1 (red fluorescence) but neither EGFP nor luc. However, upon transfection with a Cre-expression vector, they will exhibit EGFP (green fluorescence), because the loxP-flanked sequence in the integrated pCRTEIL transgenes is removed by transiently-expressed Cre protein. Thus, the behavior of descendants of the cells exhibiting Cre-mediated gene activation, which will exhibit EGFP continuously, can be monitored in real-time in vivo and in vitro.

\section{Materials and Methods}

2.1. Plasmid Construction. The reporter plasmid pCRTEIL (Figure 1(a)) was constructed through several cloning steps. First, pCRT-17, an intermediate product for pCRTEIL, was constructed by inserting an $8.8-\mathrm{kb}$ fragment containing HcRed 1 cDNA + poly(A) sites of the SV40 gene isolated from pHcRed1-N1 (Clontech Laboratories, Inc., Palo Alto, Calif, USA) in front of the $5^{\prime}$ end of CAT in the pCAG-CAT-lacZ [4] from which the lacZ gene had already been removed. A DNA fragment containing EGFP cDNA, IRES, and luc cDNA was then placed immediately downstream of the loxP site located at the $3^{\prime}$ end of the CAT gene in pCRT-17 to obtain pCRTEIL-6. The resulting pCRTEIL has a backbone of pBluescript SK(-) (Stratagene, La Jola, Calif, USA). The reporter plasmid pCETRIZ (Figure 1(a)) was constructed in a multistep process. First, pACS, an intermediate product carrying "I-Sce I-Sfi I-Xba I-Spe I-Sty I-Nhe I-Sfi I-I-Sce I" cassette, was generated by introducing linker oligo into a pBluescript II-based vector. A fragment containing IRES, lacZ gene, and poly(A) sites was introduced into a Sty I site (which had been blunt) of pACS to generate pACSIZA. Next, pCETIZ was generated by ligation of a 4.5-kb Spe I fragment containing CAG promoter, floxed EGFP cDNA and CAT gene, and poly(A) sites into an Xba I site of pACSIZA. Finally,
pCETRIZ was constructed by introducing PCR-amplified HcRed1 cDNA into a Spe I site of pCETIZ.

For expression of Cre, we used pCAG/NCre plasmid ([7]; see Figure 1(b)). pCE-29 plasmid [8] was also used as a positive control vector for expression of EGFP in murine cells (Figure 1(b)). pCL was constructed by inserting luc cDNA downstream of the CAG promoter (Figure 1(b)) and used as a positive control vector for expression of luc.

2.2. Cell Line and Transfection. NIH3T3 cells were first seeded onto gelatin-coated 6-well dishes (number 4810020; Iwaki Glass Co., Tokyo, Japan) at a density of $10^{6}$ cells/well one day before transfection and grown in Dulbecco's modified Eagle's medium (DMEM; Invitrogen Co., Carlsbad, Calif, USA) supplemented with 10\% fetal bovine serum (FBS; Invitrogen Co.) at $37^{\circ} \mathrm{C}$ in an atmosphere of $5 \% \mathrm{CO}_{2}$ in air at $37^{\circ} \mathrm{C}$. For transfection of a single plasmid, four $\mu \mathrm{g}$ of plasmid DNA was mixed with $8 \mu \mathrm{l}$ of LF2000 (number 11668-027; Invitrogen Co.) in Dulbecco's modified phosphate-buffered saline without $\mathrm{Ca}^{2+}$ and $\mathrm{Mg}^{2+}$, pH 7.4 (PBS(-)), and a total of $100 \mu \mathrm{l}$ solution was prepared according to the manufacturer's protocol. For cotransfection, two plasmids ( $3 \mu \mathrm{g}$ for each) were mixed with $12 \mu \mathrm{l}$ of LF2000 in PBS(-). These DNA/liposome complexes were added to the cell culture and incubated for 1 day at $37^{\circ} \mathrm{C}$. After transfection, cells were observed for fluorescence, as described in what follows.

2.3. Histological Analysis. Cells and tissue sections were observed using an Olympus BX60 fluorescence microscope (Olympus, Tokyo, Japan) with DM505 filters (BP460-490 and BA510IF; Olympus) and DM600 filters (BP545-580 and BA6101F; Olympus), which were used for EGFP and HcRed1 monitoring, respectively. For detection of fluorescence in dissected oviducts, an Olympus BX40 dissecting microscope was used. Microphotographs were taken using a digital camera (FUJIX HC-300/OL; Fujifilm, Tokyo, Japan) attached to the fluorescence microscope and printed out using a Mitsubishi digital color printer (CP700DSA; Mitsubishi, Tokyo, Japan).

2.4. In Vivo Gene Transfer. Intraoviductal injection was performed as described previously [9]. One $\mu$ l of solution containing plasmid DNA and trypan blue (TB; 0.05\% final concentration) was slowly injected with a glass micropipette, which had been attached to a mouthpiece, into the ampulla of an oviduct of B6C3F1 females (4- to 6-weekold; purchased from CLEA Japan, Inc., Tokyo, Japan). The DNA introduced per oviduct was pCE-29 $(0.2 \mu \mathrm{g})$, pCRTEIL $(0.2 \mu \mathrm{g})$, pCAG/NCre $(0.2 \mu \mathrm{g})$, pCRTEIL $(0.2 \mu \mathrm{g})$ $+\mathrm{pCAG} / \mathrm{NCre}(0.2 \mu \mathrm{g})$, or $\mathrm{pCL}(0.2 \mu \mathrm{g})$. In each transfection group, $0.02 \mu \mathrm{g}$ of phRL-SV (Promega Co., Madison, Wis, USA) was included as a control for normalization of luc activity as noted in what follows. After finishing the injection, the micropipette was rapidly removed. The oviductal regions were then subjected to in vivo EP. Eight square-wave pulses with pulse duration of 50 milliseconds and electric field intensity of $50 \mathrm{~V}$ were administered from a square-wave pulse generator (T820; BTX Genetronics, Inc., San Diego, 


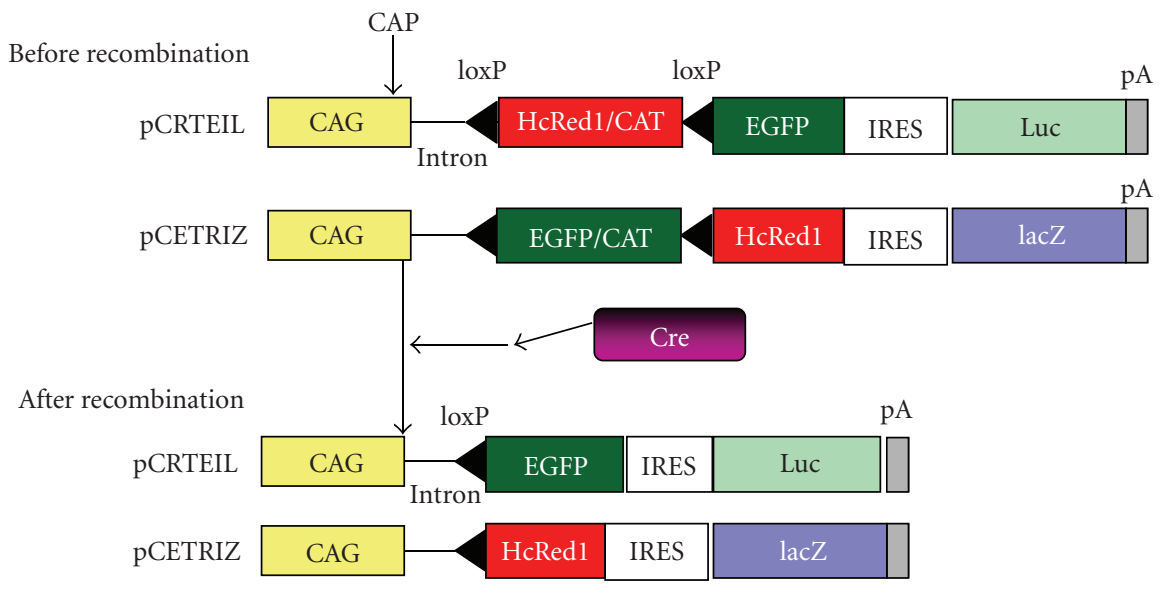

(a)

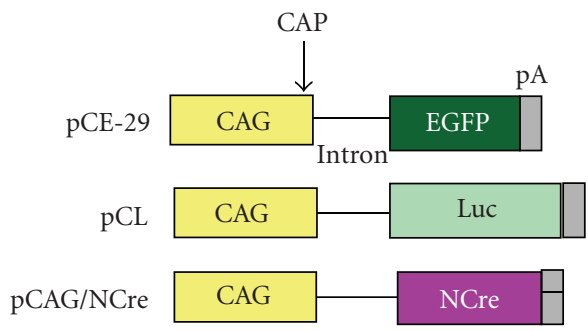

(b)

FIGURE 1: (a) Scheme for Cre-loxP-mediated recombination using pCRTEIL and pCETRIZ as reporter transgenes. Before recombination, the floxed HcRed1/CAT hybrid sequence is expressed under control of the CAG promoter in cells carrying pCRTEIL, while the EGFP and luc cDNAs are silent. Similarly, the floxed EGFP/CAT hybrid sequence is expressed in cells carrying pCETRIZ, but the HcRed1 cDNA and lacZ genes are silent. Cre-mediated recombination results in deletion of the floxed sequence, and expression of the EGFP and luc cDNAs in pCRTEIL-carrying cells or expression of HcRed 1 and lacZ in cells carrying pCETRIZ. (b) Plasmids (pCE-29, pCL, and pCAG/NCre) are used for expression of EGFP, luc, and NCre, respectively. All plasmids have a pBluescript SK(-) backbone. Abbreviations are CAG: cytomegalovirus enhancer + chicken $\beta$-actin promoter; CAP site: transcription start site; CAT: chloramphenicol acetyltransferase gene; EGFP: enhanced green fluorescent protein cDNA; IRES: internal ribosomal entry site; Luc: firefly luciferase cDNA; NCre: nuclear location signal + Cre gene; pA: poly(A) sites.

Calif, USA). One day after in vivo EP, the oviducts were subjected to observation for fluorescence and then to lysis for measurement of luc activity.

Oviducts from the mice receiving plasmid DNA were fixed in $4 \%$ paraformaldehyde (PFA) in PBS(-) for 2 days at $4^{\circ} \mathrm{C}$, then immersed in $30 \%$ sucrose in PBS(-) for more than 2 days at $4^{\circ} \mathrm{C}$, embedded in Tissue-Tek OCT compound (Sakura Finetek Co., Tokyo, Japan), and finally frozen before serial cryostat sectioning (10-30 $\mu \mathrm{m}$ in thickness). Sections were mounted in VectraShield mounting medium (Vector Laboratories, Burlingame, Calif, USA) and observed for fluorescence.

To deliver DNA-coated gold particles to the abdominal skin of adult ICR females in vivo, we used the Helios gene gun (Bio-Rad Laboratories, Hercules, Calif, USA). Before the introduction of DNA, ether-anaesthetized mice were shaved in the abdominal area. Transfection particles were made per Helios gene gun protocol giving a microcarrier loading quantity of $0.5 \mathrm{mg}$ per shot and a DNA loading ratio of 2 or $4 \mu \mathrm{g} D N A / \mathrm{mg}$ gold particles $(1.0 \mu \mathrm{m})$. For single transfection with pCETRIZ (control), $1 \mu \mathrm{g}$ of DNA was used. For cotransfection using pCETRIZ plus pCAG/NCre (experiment), $1 \mu \mathrm{g}$ of each DNA was used. Mouse abdominal skin was bombarded with the DNA-coated gold particles using the gene gun pressurized by a helium tank at 300 psi. One bombardment was carried out per mouse using 6 mice. In total, 3 transfections were performed for each group. Animals were killed at 48 hours after gene gun bombardment.

Mouse skin was excised and immediately subjected to observation for fluorescence under UV illumination. The samples were then fixed with 4\% PFA in PBS(-) for 1 day at $4^{\circ} \mathrm{C}$. Subsequently, the skin was washed in PBS(-) twice and stained with X-Gal staining solution (X-gal Staining Assay Kit, Genlantis, Calif, USA) at $30^{\circ} \mathrm{C}$ for 24 hours. The stained samples were then washed twice in PBS(-) and observed for lacZ activity under light.

2.5. Assay for Luciferase Activity. Luc assay was performed using a kit (Dual-Luciferase Reporter Assay System (number E1910); Promega Co.). The oviducts isolated 1 day after in vivo $\mathrm{EP}$ were homogenized in $1 \mathrm{~mL}$ of $1 \mathrm{X}$ reporter lysis 


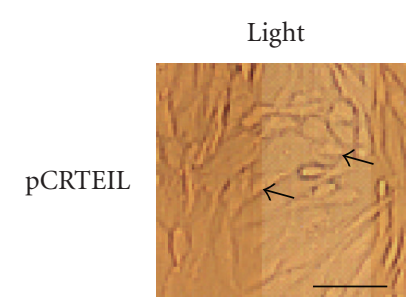

(a)

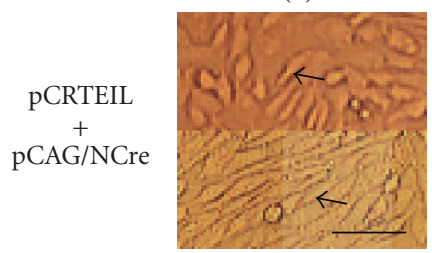

(d)

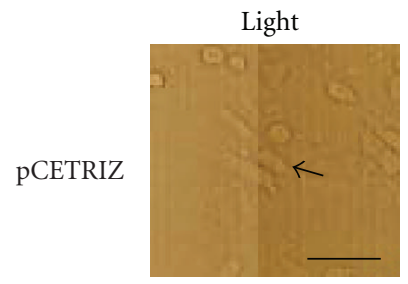

(g)

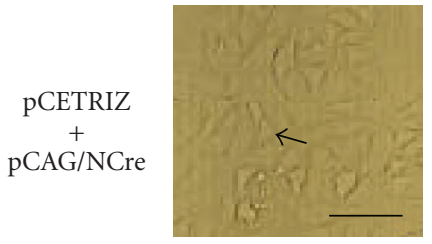

$(\mathrm{k})$

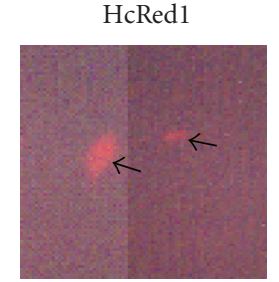

(b)

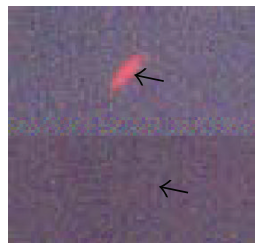

(e)

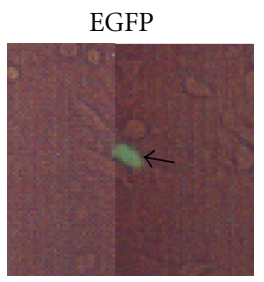

(h)

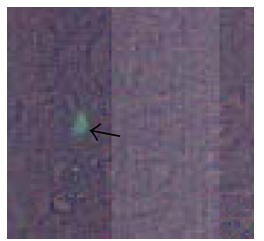

(1)

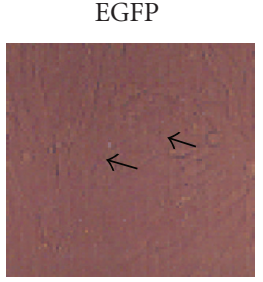

(c)

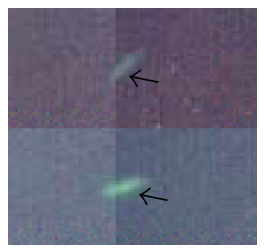

(f)

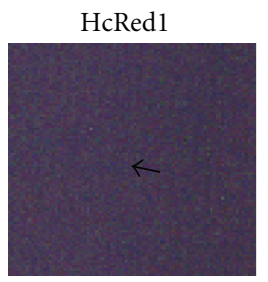

(i)

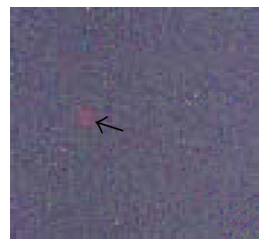

(m)

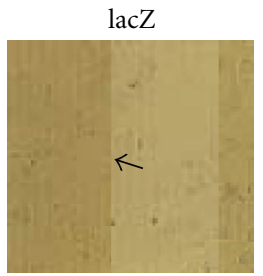

(j)

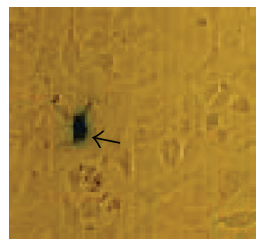

(n)

FIGURE 2: Cre-mediated excision in vitro. (a)-(c) NIH3T3 cells transfected with pCRTEIL alone. When cells were inspected for fluorescence 1 day after transfection, HcRed1-derived red fluorescence (but not EGFP-derived green fluorescence) was predominant (indicated by arrows in (b)). (a)-(c) Microphotographs indicate the same cells. (d)-(f) NIH3T3 cells cotransfected with pCRTEIL and pCAG/NCre. Some cells predominantly exhibited green fluorescence (indicated by arrows in (e) and (f)), while other cells exhibited both types of fluorescence (indicated by arrowheads in (e) and (f)). (d)-(f) Microphotographs indicate the same cells. (g)-(j) NIH3T3 cells transfected with pCETRIZ alone. When cells were inspected for fluorescence 1 day after transfection, EGFP-derived green fluorescence (but not HcRed1-derived red fluorescence and lacZ expression) was predominant (indicated by arrows in (h)). (g)-(j) Microphotographs indicate the same cells. (k)-(n) NIH3T3 cells cotransfected with pCETRIZ and pCAG/NCre. Cells exhibited both green and red fluorescence as well as lacZ expression (indicated by arrows in (l), (m), and (n)). (k)-(n) Microphotographs indicate the same cells. (a), (d), (g), and (k) Photographs taken under light (Light); (b), (e), (i), and (m) photographs taken under UV illumination using filters for detection of red fluorescence (HcRed1); (c), (f), (h), and (l) photographs taken under UV illumination using filters for detection of green fluorescence (EGFP); (j) and (n) photographs taken under light after staining with X-Gal (lacZ). Bar: $10 \mu \mathrm{m}$.

buffer (Promega Co.). After centrifugation at $15000 \mathrm{rpm}$ for 10 minutes at $4^{\circ} \mathrm{C}$, the supernatant $(200 \mu \mathrm{l})$ was transferred to a fresh Eppendorf tube. Cells 1 day after transfection were collected with a cell scraper and precipitated after brief centrifugation. Cell pellets were then lysed with $1 \mathrm{~mL}$ of $1 \mathrm{X}$ reporter lysis buffer. Relative light units (RLUs) obtained with luc were measured for 5 seconds following a 2-second delay after the addition of the lysate $(10 \mu \mathrm{l})$ to $50 \mu \mathrm{l}$ of luc assay substrate (Promega Co.) using a luminometer (number TD-20/20; Turner Designs Instrument, Sunnyvale, Calif, USA). RLUs were normalized to micrograms of tissue protein added in the luc assay. Tissue protein determinations were performed using Bradford reagent (Bio-Rad Laboratories,
Inc., Hercules, Calif, USA). At least four oviducts were tested in each transfection group, and values were presented as means \pm standard deviation (SD).

2.6. Statistical Analysis. Results are presented as means \pm SD of separate experiments. The RLU obtained from transfection into oviductal epithelium was analyzed using the SPSS 9.0/PC statistics package (SPSS Inc., Chicago, Ill, USA). Results were compared using one-way analysis of variance (ANOVA) following arcsin transformation of the proportions. The Duncan new multiple range test or Tukey test was performed if ANOVA revealed significant differences among compared values. 


\section{Results}

Successful in Vitro Cre-Mediated Recombination Results in Switching of Gene Expression. In a preliminary test, we first examined what percentage of single cells can incorporate two constructs simultaneously; NIH3T3 cells were cotransfected with pCRTEIL and pCE-29 by the lipoplex method. One day after transfection, fluorescent cells were inspected under a fluorescence microscope. Eight percent (12/142) and 10\% (14/142) of cells exhibited EGFP-derived green and HcRed1derived red fluorescence, respectively, (data not shown). Twenty-two percent (31/142) of cells expressed both types of fluorescence (data not shown). It was thus estimated that in approximately $20 \%$ of single cells, incorporation of two constructs into a single cell occurs at the same time. To test whether Cre-mediated excision of the floxed HcRed1/CAT sequence in the pCRTEIL construct results in generation of EGFP fluorescence (Figure 1(a)), NIH3T3 cells were cotransfected with pCRTEIL and pCAG/NCre by the lipoplex method. One day after transfection, some (9\% (10/112)) of these cells exhibited both red and green fluorescence (indicated by arrowheads in Figures 2(d) $-2(\mathrm{f})$ ), suggesting incomplete Cre-mediated recombination in the introduced pCRTEIL construct (Table 1). There were a few cells $(4 \%$ [4/112]) preferentially expressing green fluorescence (indicated by arrows in Figures 2(d) $-2(\mathrm{f})$ ), suggesting the occurrence of complete Cre-mediated recombination in the transfected cells (Table 1). Twenty-four percent (27/112) of cells (Table 1) expressed only red fluorescence, suggesting incorporation of two constructs at the same time in a single cell (though gene switching failed) or incorporation of pCRTEIL alone. Considering that $20 \%$ of cells can incorporate two constructs simultaneously, the rate of Cre-mediated recombination in this system appears to be greater than $50 \%$ at a minimum. In the control experiment in which cells were transfected with pCRTEIL alone, some cells (35\% (36/102); Table 1) exhibited HcRed1-derived red fluorescence but not EGFP-derived green fluorescence (arrows in Figures 2(b) versus 2(c)).

Similar results were also obtained when pCETRIZ was transfected singly or cotransfected with pCAG/NCre (Figures $2(\mathrm{~g})-2(\mathrm{n}))$. In this system, expression of lacZ protein arises from the recombined form of pCETRIZ together with HcRed1 after Cre-mediated recombination. In fact, cells expressing HcRed 1 exhibited lacZ, which could be visualized after fixation of cells and subsequent staining with X-Gal (arrows in Figures 2(k)-2(n)). Transfection of cells with a single pCETRIZ resulted in expression of EGFP but not of both HcRed1 and lacZ (arrows in Figures $2(\mathrm{~g})-2(\mathrm{j})$ ). These findings indicate that pCRTEIL and pCETRIZ as reporter plasmids work well with cultured murine cells.

Successful in Vivo Cre-Mediated Recombination in pCRTEIL System. We next examined whether this Cre-loxP system using pCRTEIL as a reporter plasmid can also be used in vivo. For this purpose, gene delivery into oviductal epithelium was performed by DNA injection into the lumen of oviducts and subsequent EP [9]. Instillation of pCE29 plasmid yielded bright green fluorescence throughout the ampulla ((b) in Figure 3(A)) but not red fluorescence ((c) in Figure $3(\mathrm{~A}))$. When pCRTEIL plasmid DNA was singly introduced, no fluorescence for EGFP was observed $((\mathrm{e})$ in Figure 3(A)). Instead, red fluorescence was observed in some oviductal epithelial cells ((f) in Figure 3(A)). Coinjection with pCRTEIL and pCAG/NCre plasmids resulted in generation of both red and green fluorescence, although green fluorescence appeared to be weaker than red fluorescence (indicated by arrows in (h) and (i) in Figure 3(A)).

To examine the localized expression of EGFP and HcRed 1 in the oviducts after cotransfection, cryostat sections were prepared and inspected for EGFP and red fluorescence. Strong, but patchy, EGFP fluorescence was observed in some oviductal epithelial cells (indicated by arrows in (k) in Figure 3(A)), together with weak red fluorescence throughout the EGFP-positive area (data not shown). These findings indicate that the Cre-loxP system using pCRTEIL works well even in vivo, and as expected HcRed 1 exhibits a nonoverlapping spectral profile from EGFP.

The IRES sequence is present between EGFP and luc cDNAs in the pCRTEIL plasmid (Figure 1(a)). Therefore, the two proteins (EGFP and luc) should be produced at the same time in a cell into which pCRTEIL and pCAG/NCre have been cotransfected, since an IRES sequence allows cap-independent translation initiation $[10,11]$ from the dicistronic mRNA generated. In fact, we observed increase in luc activity repeatedly when oviductal epithelial cells were cotransfected with pCRTEIL and pCAG/NCre (Figure 3(B)). This finding also suggests successful in vivo Cre-mediated recombination.

Successful in Vivo Cre-Mediated Recombination in pCETRIZ System. Cells carrying pCETRIZ are expected to exhibit HcRed 1 and lacZ after Cre-mediated excision. For testing the feasibility of pCETRIZ-based Cre-loxP system in vivo, tissues that never exhibit endogenous $\beta$-galactosidase activity must be chosen. In this occasion, skin epidermis appeared to be suitable for this purpose, because it does not exhibit such kind of activity [12] and has been considered as an attractive target to study gene delivery due to its easy accessibility and visualization [13]. Since particle-mediated devices have been studied to deliver genes to skin efficiently $[12,14$, 15], we introduced pCETRIZ (control) or pCETRIZ + pCAG/NCre (experiment) into mouse skin using Helios gene gun. Skin biopsies were taken after 48 hours for inspection of fluorescence and lacZ staining. In the samples bombarded with single pCETRIZ, green fluorescence was detected over the area gene-transfected (Figure 4(b)). However, neither red fluorescence nor lacZ activity was noted in the area exhibiting green fluorescence (Figures 4(c) and 4(d)). This was also observed in the other 2 injected mice. Samples bombarded with pCETRIZ + pCAG/NCre showed coexpression of green and red fluorescence (arrows in Figures 4(f) and $4(\mathrm{~g})$ ), suggesting successful Cre-mediated excision of the introduced pCETRIZ. As expected, lacZ staining revealed expression of lacZ over the area cotransfected (Figure 4(h)). These patterns of gene expression were also seen in the other 2 injected mice. 


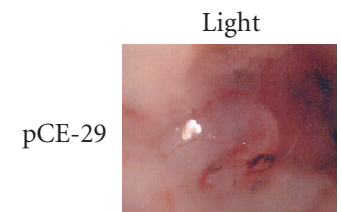

(a)

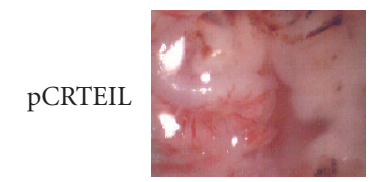

(d)

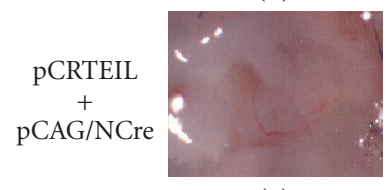

(g)

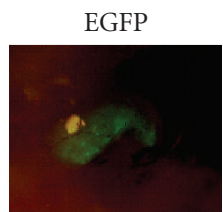

(b)

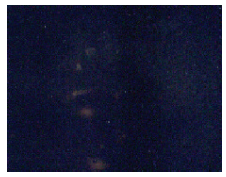

(e)

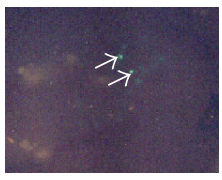

(h)

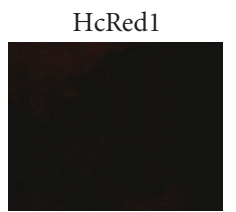

(c)

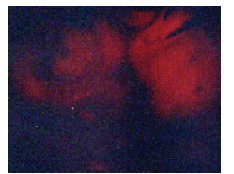

(f)

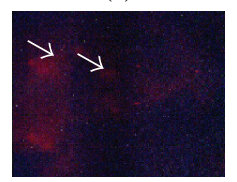

(i)

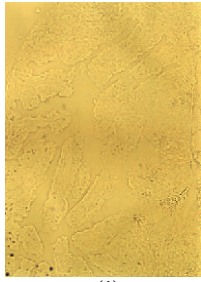

(j)

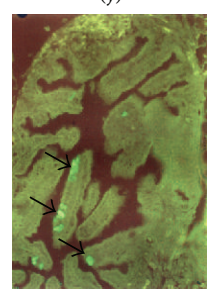

(k)

(A)

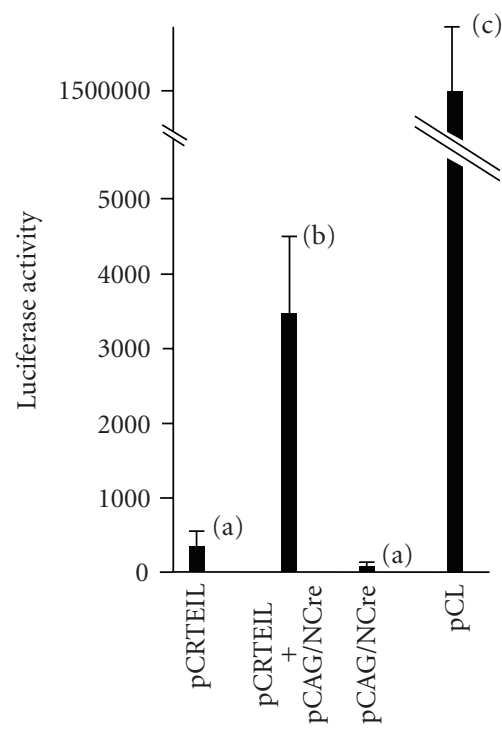

(B)

FIGURE 3: Cre-mediated excision in vivo. (A): (a)-(c) Oviducts transfected with pCE-29 alone. EGFP-derived green fluorescence (but not HcRed1-derived red fluorescence) was observed. (d)-(f) Oviducts transfected with pCRTEIL alone. HcRed1-derived red fluorescence (but not EGFP-derived green fluorescence) was observed. (g)-(i) Oviducts cotransfected with pCRTEIL and pCAG/NCre. Both types of fluorescence (indicated by arrows in (h) and (i)) were seen, although green fluorescence appeared to be weaker than red fluorescence. (j) and (k) Cryostat sections of oviducts sampled 1 day after introduction of pCRTEIL and pCAG/NCre DNA $(0.2 \mu \mathrm{g}$ each) and subsequent in vivo electroporation. Note that strong, but patchy, EGFP fluorescence presents in some oviductal epithelial cells (indicated by arrows in (k)). (a), (d), (g), and (j) Photographs taken under light (Light); (b), (e), (h), and (k): photographs taken under UV illumination using filters for detection of green fluorescence (EGFP); (c), (f), and (i) photographs taken under UV illumination using filters for detection of red fluorescence (HcRed1). (B) Luciferase reporter gene activity in oviduct sampled 1 day after in vivo gene delivery to oviducts. Oviducts were in vivo transfected with pCRTEIL alone (negative control), pCRTEIL and pCAG/NCre (experiment), pCAG/NCre alone (negative control), or pCL (positive control). Oviducts were isolated from each mouse at 2 days following electroporation to measure luc activity. From a total of 8 females injected with DNA, 4 oviducts were subjected to measurement of luc activity for each transfection group. Note also that the vertical axis of the figure is interrupted and has one scale. Different letters indicate statistically significant differences $(P<.001)$.

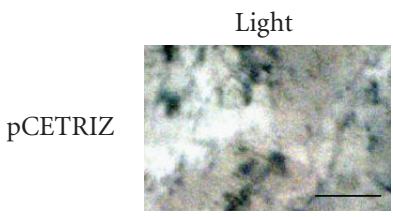

(a)

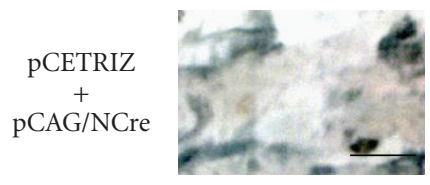

(e)

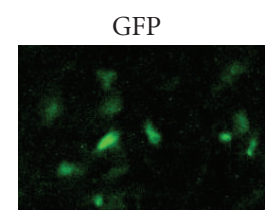

(b)

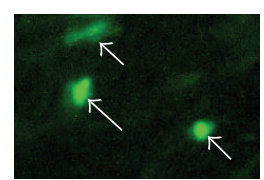

(f)

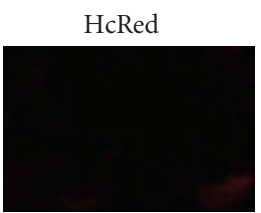

(c)

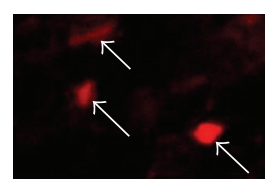

(g)

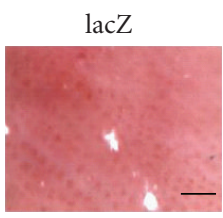

(d)

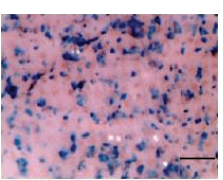

(h)

FIGURE 4: Gene gun-mediated skin transfection with pCETRIZ (a)-(d) and pCETRIZ + pCAG/NCre (e)-(h). After shaving of the abdominal area of mice, plasmid DNA coated with gold particles was transfected with a 300-psi helium gas pulse using the Helios gene gun. Two days after gene delivery, skin was dissected and inspected for fluorescence under a UV illuminator. After observation for fluorescence, the same sample was then subjected to histochemical staining for lacZ activity. Figures (a)-(c) and (e)-(g) are photographs of the same portion. Figure (d) is a different portion from those in Figures (a)-(c). Similarly, Figure (h) is a different portion from those in Figures (e)-(g). (a), (d), (e), and (h) taken under light; (b) and (f): taken under UV illumination for detection of GFP fluorescence; (c) and (g): taken UV illumination for detection of red fluorescence. Bar: $10 \mu \mathrm{m}$. 
TABle 1: Summary of efficiency of Cre-mediated recombination in NIH3T3 cells after cotransfection with tester and Cre-expression constructs.

\begin{tabular}{|c|c|c|c|c|}
\hline & & No gene switching ${ }^{1}$ & $\begin{array}{l}\text { Complete gene } \\
\text { switching }\end{array}$ & $\begin{array}{l}\text { Incomplete gene } \\
\text { switching }{ }^{1}\end{array}$ \\
\hline $\begin{array}{l}\text { Plasmid } \\
\text { construct(s) } \\
\text { used }\end{array}$ & $\begin{array}{l}\text { Total number } \\
\text { of cells } \\
\text { examined }\end{array}$ & $\begin{array}{c}\text { No. of cells expressing only } \\
\text { HcRed1-derived } \\
\text { fluorescence }(\%)\end{array}$ & $\begin{array}{l}\text { No. of cells expressing } \\
\text { only EGFP-derived } \\
\text { fluorescence }(\%)\end{array}$ & $\begin{array}{c}\text { No. of cells expressing } \\
\text { both types of } \\
\text { fluorescence }(\%)\end{array}$ \\
\hline pCRTEIL & 102 & $36(35)$ & $0(0)$ & $0(0)$ \\
\hline $\begin{array}{l}\text { pCRTEIL + } \\
\text { pCAG/NCre }\end{array}$ & 112 & $27(24)$ & $4(4)$ & $10(9)$ \\
\hline
\end{tabular}

${ }^{1}$ Cells one day after transfection were observed for fluorescence under a fluorescence microscope, and regions containing fluorescent and neighboring nonfluorescent cells were photographed. The fluorescent cells were classified into groups with no gene switching (cells exhibiting only red fluorescence; as exemplified by the cells (arrowed) in Figure 2(b)), complete gene switching (cells exhibiting only green fluorescence; as exemplified by the cell (indicated by arrowheads) in Figures 2(e), 2(f)), or incomplete gene switching (cells exhibiting both fluorescence; as exemplified by the cell (arrowed) in Figures 2(e), 2(f)). Regions comprising 30-40 cells were photographed, and fluorescent cells were scored. The numbers of cells in a total of 3 regions are listed in the table.

\section{Discussion}

The Cre-loxP system permits the generation of mouse models, in which the fate of a cell can be followed through time. In such models, a cell-type-specific promoter that is turned on at a given stage of differentiation regulates Cre-expression. When combined with a reporter system, regulated expression of Cre may lead to specific expression of inductive reporter molecules and permanent tagging of those cells, which have completed a defined genetic program. An essential component of such strategy is the development of appropriate reporter strains of mice in which the inducible reporter molecule is ubiquitously expressed.

Several reporter strains carrying different floxed transgenes and exhibiting switching of gene expression upon Cremediated recombination have been reported in mice (i.e., CAG-CAT-Z [4], Rosa26R (R26R) [16], Z/AP [17], Z/EG [18], ROSA26flox [19], CMV-floxed stop-lacZ [20], ACZL [21], and CETZ-17 [6]). Almost all have employed a single fluorescent marker gene, alkaline phosphatase, (ALP) and/or lacZ gene. The most commonly utilized strain appears to be Rosa26R mice [16], which are generated by targeting a reporter construct to the proviral ROSA26 locus that is constitutively expressed in all tissues. In these reporter strains carrying lacZ or ALP, detection of these reporters following Cre-mediated recombination should always be performed for fixed samples, thus hampering continuous observation of activated gene products in living cells or embryos. There are only a few reports on reporter strains capable of exhibiting noninvasive fluorescent markers upon Cre-mediated excision of a "stop sequence." Such strains carry a single fluorescent marker (EGFP for Schwenk et al. [22], Mao et al. [23], Motoike et al. [24], and Kawamoto et al. [25], DsRed-T3 for Vintersten et al. [26], and ECFP or EYFP for Srinivas et al. [27]), placed downstream of the floxed stop sequence. In our CRTEIL system, the reporter plasmid contains two fluorescent markers (i.e., floxed HcRed1 cDNA and EGFP placed downstream of the floxed gene) enabling monitoring of gene switching based on changes from red to green fluorescence (see Figure 1(a)). This type of Cre excision reporter construct was first reported by Yang and Hughes [28], who adopted two fluorescent markers (EGFP and
DsRed1 in their reporter plasmid, called Cre stoplight) and demonstrated that cells carrying Cre stoplight as transgene can be converted from red to green by transient expression of Cre. However, DsRed1 protein has displayed toxicity in murine embryos [29]. For these reasons, we used HcRed, a dimeric far-red fluorescent protein variant [30] isolated from the coral reef Heteractis crispa, which exhibits a spectral profile (exc. $588 \mathrm{~nm}$, em. $618 \mathrm{~nm}$ ) that does not overlap with those of other existing fluorescent proteins. HcRed 1 has already been used as a safe noninvasive protein marker in a transgenic system [31].

Recently, Muzumdar et al. [32] generated $\mathrm{mT} / \mathrm{mG}$; a double-fluorescent Cre reporter mouse that expresses membrane-targeted tandem dimer Tomato $(\mathrm{mT})$ prior to Cre-mediated excision and membrane-targeted green fluorescent protein $(\mathrm{mG})$ after excision. The use of a doublefluorescent marker system by Muzumdar et al. [32] is the same as ours. They observed that this strain works well in vivo after mating with the pre-existing Cre expressor strains. This encouraged us to generate transgenic mouse strains carrying CRTEIL/CETRIZ transgenes. The advantage of our system over that of Muzumdar et al. [32], may be the employment of IRES-luc and IRES-lacZ. These elements will be useful when tissue samples are after Cre-mediated excision subjected to biochemical and histochemical analyses, since we can measure the level of luc or lacZ quantitatively using commercially available kits (e.g., the Luminescent $\beta$ galactosidase Detection Kit II (Clontech Laboratories, Inc., Palo Alto, Calif, USA)) after observation for fluorescence. In this study, ca. 630-bp IRES placed between EGFP and luc sequences was useful for simultaneous expression of the two proteins, though the level of luc placed downstream of the IRES appeared to be greatly decreased (approximately $1 / 400$ of luc activity in the oviducts transfected with pCL alone; see Figure 3(B)). This appears to be due to the insertion of IRES between EGFP and luc, since the level of luc in the oviducts transfected with pCTL (composed of CAG promoter, loxP-flanked CAT sequence, and luc gene) + pTC (carrying an NCre gene controlled by Herpes simplex virusderived thymidine kinase promoter) was only $1 / 4$ of that in the oviducts transfected with pCL alone [33]. In this sense, use of 81-bp 2A sequence, which is known to yield synthesis 
of two different proteins from a single mRNA [34, 35], may be appropriate in our system, since similar levels of each of the proteins have been reported to be produced in cells transfected with the 2A-containing construct [36].

In this study, we have described in vitro and in vivo switching of gene expression from red to green or green to red fluorescence by the Cre-loxP system when pCRTEIL and pCETRIZ plasmids are used as tester plasmids. These events occurred when tester plasmid and Cre-expression plasmid are cotransfected (see Figures 2-4). In our LF2000-based transfection system, approximately $20 \%$ of NIH3T3 cells could incorporate two constructs simultaneously, of which more than $50 \%$ exhibited Cre-mediated gene switching. This rate appears to be too low, since in our previous experiments using F9 embryonal carcinoma cell line stably transfected with loxP-containing reporter plasmid pCETZ-17, all clones exhibited gene switching within one day after transient expression of pCAG/NCre [6]. This means that 100\% gene switching is possible if stable cell lines carrying a reporter construct are subjected to Cre-mediated transfection. The low efficiency in Cre-mediated recombination in the present study may be due solely to the cotransfection method itself, since we cannot strictly control the number of copies of exogenous gene introduced into cells.

The present Cre-loxP system based on two fluorescent markers appeared to have great advantages over the previous systems, since the process of change in gene expression can be easily monitored in noninvasive fashion. Furthermore, it is possible to trace the fate of the descendants that express fluorescence under physiological conditions. For finer analysis of the fate of cells transfected with exogenous Cre-expression vector, it will be convenient to use transgenic mouse lines carrying the CRTEIL or pCETRIZ transgene as generalized reporter strains. This experiment is now underway.

\section{Acknowledgments}

The authors would like to thank Dr. Kimi Araki (Kumamoto University, Japan) for allowing them to use pCAG-CAT-Z plasmid. They also thank Kazunori Kiryu and Masaki Takeda for their support on cryostat-sectioning. This study was supported by Grants-in-Aid from the Ministry of Education, Culture, Sports, Science and Technology, Japan.

\section{References}

[1] N. Sternberg and D. Hamilton, "Bacteriophage P1 site-specific recombination. I. Recombination between loxP sites," Journal of Molecular Biology, vol. 150, no. 4, pp. 467-486, 1981.

[2] R. H. Hoess, M. Ziese, and N. Sternberg, "P1 site-specific recombination: nucleotide sequence of the recombining sites," Proceedings of the National Academy of Sciences of the United States of America, vol. 79, no. 11, pp. 3398-3402, 1982.

[3] A. Porter, "Controlling your losses: conditional gene silencing in mammals," Trends in Genetics, vol. 14, no. 2, pp. 73-79, 1998.

[4] K. Araki, M. Araki, J.-I. Miyazaki, and P. Vassalli, "Site-specific recombination of a transgene in fertilized eggs by transient expression of Cre recombinase," Proceedings of the National
Academy of Sciences of the United States of America, vol. 92, no. 1, pp. 160-164, 1995.

[5] H. Niwa, K. Yamamura, and J. Miyazaki, "Efficient selection for high-expression transfectants with a novel eukaryotic vector," Gene, vol. 108, no. 2, pp. 193-200, 1991.

[6] M. Sato, Y. Yasuoka, H. Kodama, T. Watanabe, J.-I. Miyazaki, and M. Kimura, "New approach to cell lineage analysis in mammals using the Cre-loxP system," Molecular Reproduction and Development, vol. 56, no. 1, pp. 34-44, 2000.

[7] M. Sato, T. Watanabe, and M. Kimura, "Embryo transfer via oviductal wall: an alternative method for efficient production of transgenic mice," Transgenics, vol. 2, no. 4, pp. 383-389, 1999.

[8] M. Sato, A. Ishikawa, and M. Kimura, "Direct injection of foreign DNA into mouse testis as a possible in vivo gene transfer system via epididymal spermatozoa," Molecular Reproduction and Development, vol. 61, no. 1, pp. 49-56, 2002.

[9] M. Sato, "Intraoviductal introduction of plasmid DNA and subsequent electroporation for efficient in vivo gene transfer to murine oviductal epithelium," Molecular Reproduction and Development, vol. 71, no. 3, pp. 321-330, 2005.

[10] D. G. Kim, H. M. Kang, S. K. Jang, and H.-S. Shin, "Construction of a bifunctional mRNA in the mouse by using the internal ribosomal entry site of the encephalomyocarditis virus," Molecular and Cellular Biology, vol. 12, no. 8, pp. 36363643, 1992.

[11] P. Mountford, B. Zevnik, A. Düwel, et al., "Dicistronic targeting constructs: reporters and modifiers of mammalian gene expression," Proceedings of the National Academy of Sciences of the United States of America, vol. 91, no. 10, pp. 4303-4307, 1994.

[12] M. T. S. Lin, F. Wang, J. Uitto, and K. Yoon, "Differential expression of tissue-specific promoters by gene gun," British Journal of Dermatology, vol. 144, no. 1, pp. 34-39, 2001.

[13] M. T. S. Lin, L. Pulkkinen, J. Uitto, and K. Yoon, "The gene gun: current applications in cutaneous gene therapy," International Journal of Dermatology, vol. 39, no. 3, pp. 161$170,2000$.

[14] W. H. Sun, J. K. Burkholder, J. Sun, et al., "In vivo cytokine gene transfer by gene gun reduces tumor growth in mice," Proceedings of the National Academy of Sciences of the United States of America, vol. 92, no. 7, pp. 2889-2893, 1995.

[15] V. Staniforth, S.-Y. Wang, L.-F. Shyur, and N.-S. Yang, "Shikonins, phytocompounds from Lithospermum erythrorhizon, inhibit the transcriptional activation of human tumor necrosis factor alpha promoter in vivo," The Journal of Biological Chemistry, vol. 279, no. 7, pp. 5877-5885, 2004.

[16] P. Soriano, "Generalized lacZ expression with the ROSA26 Cre reporter strain," Nature Genetics, vol. 21, no. 1, pp. 70-71, 1999.

[17] C. G. Lobe, K. E. Koop, W. Kreppner, H. Lomeli, M. Gertsenstein, and A. Nagy, "Z/AP, a double reporter for Cremediated recombination," Developmental Biology, vol. 208, no. 2, pp. 281-292, 1999.

[18] A. Novak, C. Guo, W. Yang, A. Nagy, and C. G. Lobe, “Z/EG, a double reporter mouse line that expresses enhanced green fluorescent protein upon Cre-mediated excision," Genesis, vol. 28, no. 3-4, pp. 147-155, 2000.

[19] X. Mao, Y. Fujiwara, and S. H. Orkin, "Improved reporter strain for monitoring Cre recombinase-mediated DNA excisions in mice," Proceedings of the National Academy of Sciences of the United States of America, vol. 96, no. 9, pp. 5037-5042, 1999. 
[20] L. St-Onge, P. A. Furth, and P. Gruss, "Temporal control of the Cre recombinase in transgenic mice by a tetracycline responsive promoter," Nucleic Acids Research, vol. 24, no. 19, pp. 3875-3877, 1996.

[21] K. Akagi, V. Sandig, M. Vooijs, et al., "Cre-mediated somatic site-specific recombination in mice," Nucleic Acids Research, vol. 25, no. 9, pp. 1766-1773, 1997.

[22] F. Schwenk, U. Baron, and K. Rajewsky, "A cre-transgenic mouse strain for the ubiquitous deletion of loxP-flanked gene segments including deletion in germ cells," Nucleic Acids Research, vol. 23, no. 24, pp. 5080-5081, 1995.

[23] X. Mao, Y. Fujiwara, A. Chapdelaine, H. Yang, and S. H. Orkin, "Activation of EGFP expression by Cre-mediated excision in a new ROSA26 reporter mouse strain," Blood, vol. 97, no. 1, pp. 324-326, 2001.

[24] T. Motoike, S. Loughna, E. Perens, et al., "Universal GFP reporter for the study of vascular development," Genesis, vol. 28 , no. 2 , pp. $75-81,2000$.

[25] S. Kawamoto, H. Niwa, F. Tashiro, et al., "A novel reporter mouse strain that expresses enhanced green fluorescent protein upon Cre-mediated recombination," FEBS Letters, vol. 470, no. 3, pp. 263-268, 2000.

[26] K. Vintersten, C. Monetti, M. Gertsenstein, et al., "Mouse in red: red fluorescent protein expression in mouse ES cells, embryos, and adult animals," Genesis, vol. 40, no. 4, pp. 241246, 2004.

[27] S. Srinivas, T. Watanabe, C.-S. Lin, et al., "Cre reporter strains produced by targeted insertion of EYFP and ECFP into the ROSA26 locus," BMC Developmental Biology, vol. 1, article 4, pp. 1-8, 2001.

[28] Y. S. Yang and T. E. Hughes, "Cre stoplight: a red/green fluorescent reporter of Cre recombinase expression in living cells," BioTechniques, vol. 31, no. 5, pp. 1036-1041, 2001.

[29] A. K. Hadjantonakis, S. Macmaster, and A. Nagy, "Embryonic stem cells and mice expressing different GFP variants for multiple non-invasive reporter usage within a single animal," BMC Biotechnology, vol. 2, article 11, pp. 1-9, 2002.

[30] N. G. Gurskaya, A. F. Fradkov, A. Terskikh, et al., "GFP-like chromoproteins as a source of far-red fluorescent proteins," FEBS Letters, vol. 507, no. 1, pp. 16-20, 2001.

[31] H. Hasuwa, K. Kaseda, T. Einarsdottir, and M. Okabe, "Small interfering RNA and gene silencing in transgenic mice and rats," FEBS Letters, vol. 532, no. 1-2, pp. 227-230, 2002.

[32] M. D. Muzumdar, B. Tasic, K. Miyamichi, N. Li, and L. Luo, "A global double-fluorescent Cre reporter mouse," Genesis, vol. 45, no. 9, pp. 593-605, 2007.

[33] S. Nakamura, S. Watanabe, M. Ohtsuka, et al., "Cre-loxP system as a versatile tool for conferring increased levels of tissue-specific gene expression from a weak promoter," Molecular Reproduction and Development, vol. 75, no. 6, pp. 1085-1093, 2008.

[34] M. L. L. Donnelly, G. Luke, A. Mehrotra, et al., "Analysis of the aphthovirus $2 \mathrm{~A} / 2 \mathrm{~B}$ polyprotein 'cleavage' mechanism indicates not a proteolytic reaction, but a novel translational effect: a putative ribosomal 'skip," Journal of General Virology, vol. 82, no. 5, pp. 1013-1025, 2001.

[35] M. L. L. Donnelly, L. E. Hughes, G. Luke, et al., “The 'cleavage' activities of foot-and-mouth disease virus 2A site-directed mutants and naturally occurring '2A-like' sequences,' Journal of General Virology, vol. 82, no. 5, pp. 1027-1041, 2001.

[36] K. Hasegawa, A. B. Cowan, N. Nakatsuji, and H. Suemori, "Efficient multicistronic expression of a transgene in human embryonic stem cells," Stem Cells, vol. 25, no. 7, pp. 1707 1712, 2007. 

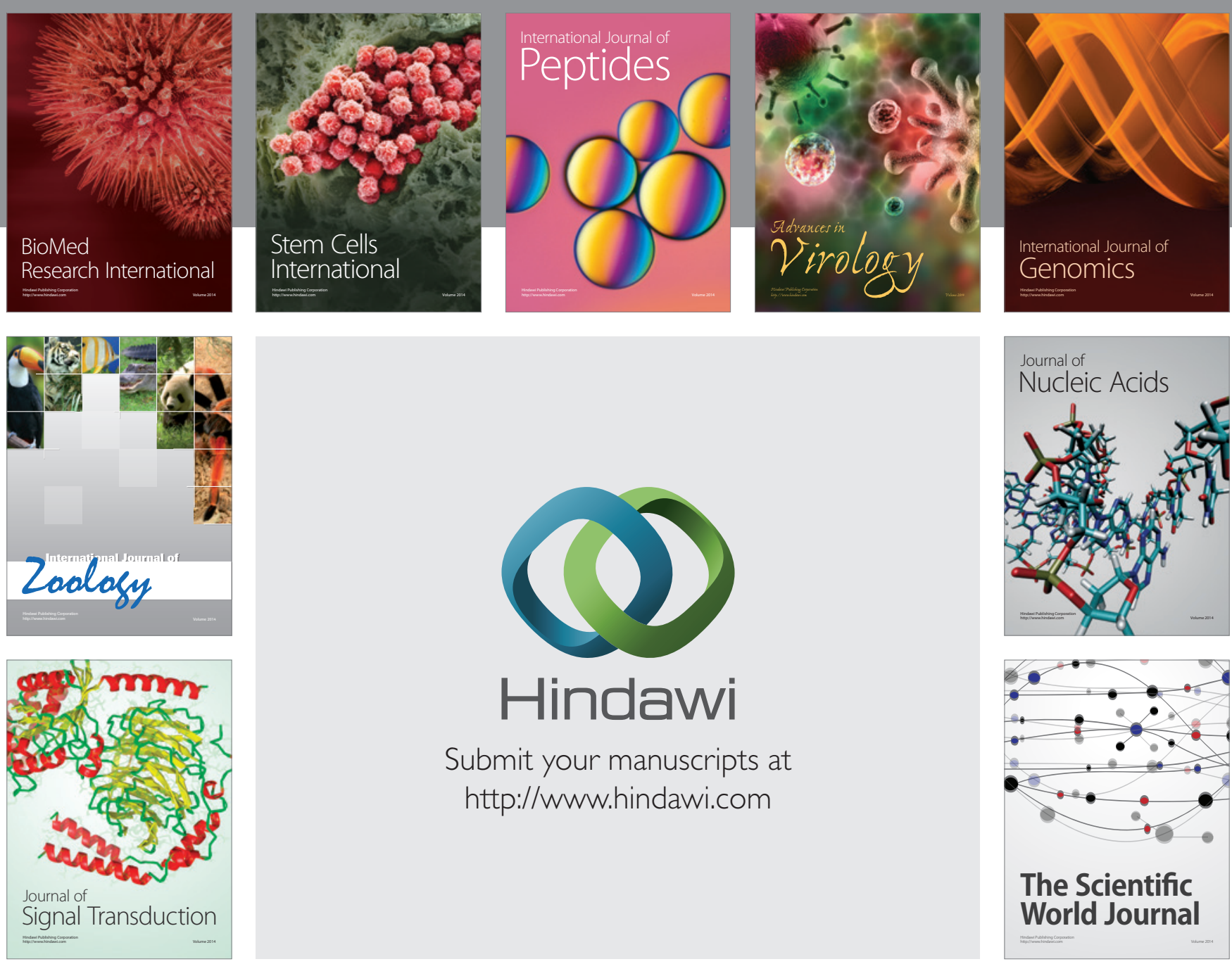

Submit your manuscripts at

http://www.hindawi.com
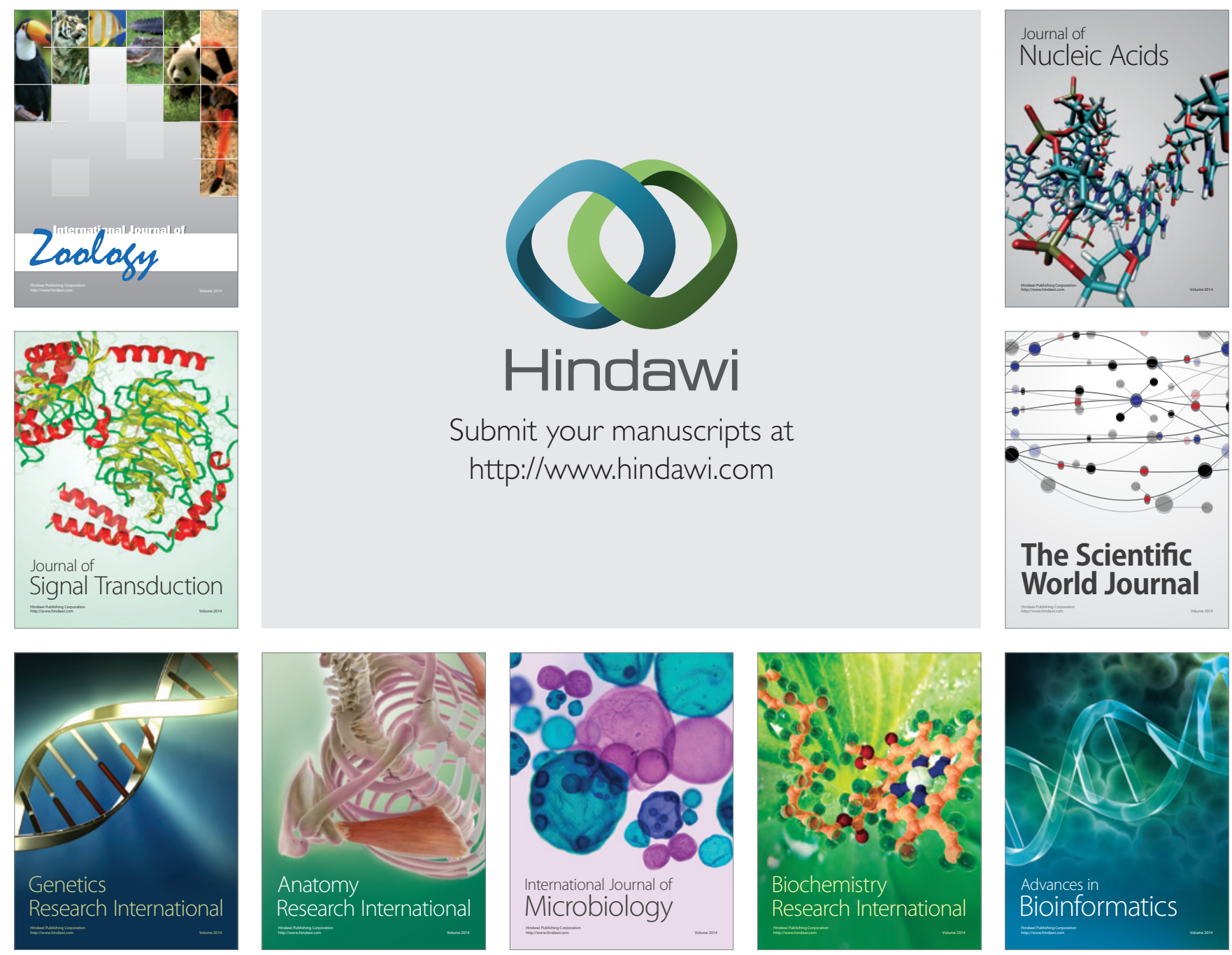

The Scientific World Journal
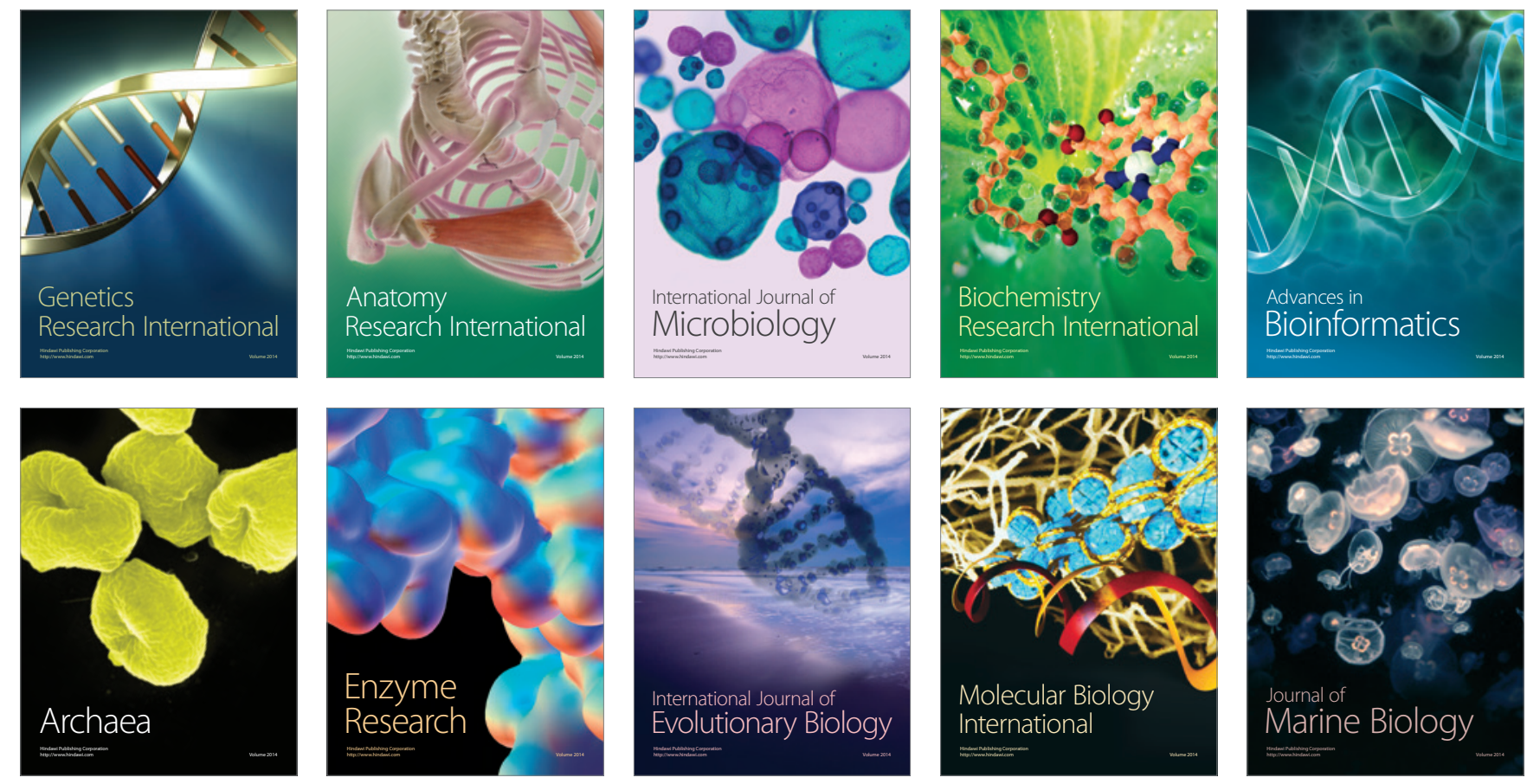